\title{
Prevalence and determinants of antenatal depression in Ethiopia: A systematic review and meta-analysis
}

\author{
Getinet Ayano $^{1 *}$, Getachew Tesfaw ${ }^{1,2}$, Shegaye Shumet ${ }^{2}$ \\ 1 Research and Training Department, Amanuel Mental Specialized Hospital, Addis Ababa, Ethiopia, \\ 2 Department of Psychiatry, University of Gondar, Gondar, Ethiopia \\ * babiget2015@gmail.com
}

\section{Abstract}

\section{Background}

Maternal depression is the most prevalent psychiatric disorder during pregnancy, can alter fetal development and have a lasting impact on the offspring's neurological and behavioral development. However, no review has been conducted to report the consolidated magnitude of antenatal depression (AND) in Ethiopia. Therefore, this review aimed to systematically summarize the existing evidence on the epidemiology of AND in Ethiopia.

\section{OPEN ACCESS}

Citation: Ayano G, Tesfaw G, Shumet S (2019) Prevalence and determinants of antenatal depression in Ethiopia: A systematic review and meta-analysis. PLoS ONE 14(2): e0211764. https:// doi.org/10.1371/journal.pone.0211764

Editor: Astrid M. Kamperman, Erasmus Medical Center, NETHERLANDS

Received: December 21, 2017

Accepted: January 21, 2019

Published: February 19, 2019

Copyright: @ 2019 Ayano et al. This is an open access article distributed under the terms of the Creative Commons Attribution License, which permits unrestricted use, distribution, and reproduction in any medium, provided the original author and source are credited.

Data Availability Statement: All relevant data are within the paper and its Supporting Information file.

Funding: We confirm that no funding was received for this study.

Competing interests: The authors have declared that no competing interests exist.

\section{Methods}

Using PRISMA guideline, we systematically reviewed and meta-analyzed studies that examined the prevalence and associated factors of AND from three electronic databases (PubMed, EMBASE, and SCOPUS). We used predefined inclusion criteria to screen identified studies. A qualitative and quantitative analysis was employed. Heterogeneity across the studies was evaluated using $Q$ and the $\mathrm{I}^{2}$ test. Publication bias was assessed by funnel plot and Egger's regression test.

\section{Results}

In this review, a total of 193 studies were initially identified and evaluated. Of these, five eligible articles were included in the final analysis. In our meta-analysis, the pooled prevalence of AND in Ethiopia was 21.28\% (95\% Cl; 15.96-27.78). The prevalence of AND was highest in the third trimester of pregnancy at $32.10 \%$ and it was $19.13 \%$ in the first trimester and $18.86 \%$ in the second trimester of pregnancy. The prevalence of AND was $26.48 \%$ and $18.28 \%$ as measured by Beck depression inventory (BDI) and the Edinburgh Postnatal Depression Scale (EPDS), respectively. Moreover, the prevalence of AND was $15.50 \%$ for the studies conducted in the community setting and it was $25.77 \%$ for the studies conducted in the institution-based setting. In our qualitative synthesis, we found that those pregnant women who had a history of stillbirth, complications during pregnancy, previous history of depression, no ANC follow-up, irregular ANC follow-up, not satisfied by ANC follow-up, and monthly income $<1500$ Ethiopian birr were linked with a greater risk of developing ANC. We 
also found that those women who experienced partner violence during pregnancy, food insecurity, medium and low social support, and those who were unmarried, age group 2029 , house wives and farmers were associated with a higher risk of developing ANC.

\section{Conclusion and recommendations}

Our meta-analysis found that the pooled prevalence of AND in Ethiopia was $21.28 \%$. The prevalence of AND was high in the third trimester of pregnancy as compared to the first and second trimesters of pregnancy. The prevalence of AND was high in studies conducted using BDI than EPDS. Studies on the magnitude of AND as well as the possible determinants in each trimester of pregnancy with representative sample size are recommended. Screening of depression in a pregnant woman in perinatal setting might be considered backed by integration of family planning and mental health services. The use of validated and a standard instrument to assess AND is warranted.

\section{Systematic review registration}

The protocol for this systematic review and meta-analysis was registered at PROSPERO (record ID=CRD42017076521, 06 December 2017)

\section{Background}

Pregnancy is the period of great joy and positive expectations, but also physical and mental stress and difficulties. Pregnancy is associated with a range of physiological as well as psychological changes for the mothers and they are expected to face numerous new challenges in this period. As a result, the perinatal period is associated with a considerably greater risk of experiencing mental health problems for all women $[1,2]$. Of the varies mental health problems, maternal depression is the most prevalent psychiatric disorder during pregnancy $[1,3$, 4], can alter fetal development and have a lasting impact on the offspring's neurological and behavioral development $[5,6]$.

Epidemiologic studies showed that the estimated prevalence of antenatal depression (AND) varies between studies. The estimated prevalence of AND varies between $7 \%$ and $20 \%$ in developed countries depending on the study [7-10]. According to a recent meta-analysis, the pooled prevalence estimates of antenatal natal depression (AND) in the low and middle-income countries (LMICs) was $25.3 \%$ (95\% CI 21.4-29.6\%) [11], whereas, the reported prevalence estimates of AND ranges between $4 \%$ and $56 \%[1,3,5,12-22]$. The type of the instrument used to assess depression, the study setting, the trimester of pregnancy, the socioeconomic and cultural differences are among the common possible reasons for the observed variation in the magnitude of AND [1, 3, 5, 12-22].

Scientific studies also found that AND commonly precedes postnatal depression [23] and associated with a higher risk of suffering to the woman as well as her family[24]. In addition, it has been shown that AND is among the leading causes of perinatal morbidity and mortality in women [25].

The evidence from the reported prevalence estimate of AND shows that the prevalence of AND was high in LMICs, such as, 20.2 \% in Brazil [26], 25 \% in Pakistan[27], $29 \%$ in Bangladesh [28], 39 \% in South Africa Cape Town, 38.5 \%[29] in South Africa KwaZulu-Natal[30] 
and $39.5 \%$, in Tanzania[31]. The estimated prevalence of AND in Ethiopia ranges between $11.8 \%$ and $31.1 \%$ depending on the study [32-36].

Moreover, the estimated prevalence of AND varies depending on the different trimesters of pregnancy. For example, according to the results of a meta-analysis conducted in the LMICs, the magnitude of AND was $7.4 \%(2.2-12.6 \%)$ in the first trimester, and it was $12.8 \%$ (10.7$14.8 \%)$ in the second trimester and $12.0 \%(7.4-16.7 \%)$ in the third trimester of pregnancy [1].

Epidemiological studies has identified several factors that were responsible for an increased risk of AND such as, younger age, unplanned pregnancy, unmarried by marital state, lack of family support, lack of spouse support, past history of psychiatric disorders, violence during pregnancy, low socioeconomic status, stressful life events, first pregnancy, poor antenatal care follow up, pregnancy-related complications and previous operative delivery [32-37].

Even though we found a remarkable variation in the magnitude of AND among the studies conducted in Ethiopia in addition to the observed methodological weakness associated to the measurement and substantial inconsistency across the findings, there are no previous systematic review and meta-analysis studies conducted in Ethiopia on the prevalence and determinates of AND. Therefore, the objective of this study was to perform a systematic review of studies conducted in Ethiopia on prevalence and predisposing factors of AND and to systematically summarize: (1) the prevalence of AND (2) the prevalence of AND during the three trimesters of pregnancy (3) the determinants of AND, and to formulate recommendations for future research.

\section{Methods/design}

This systematic review and meta-analysis was conducted following "the Preferred Reporting Items for Systematic Reviews and Meta-Analyses (PRISMA)" guidelines [38]. We conducted an extensive search of the literature in three databases (EMBASE, MEDLINE, and Scopus). The following terms and keywords were applied for PubMed search: (prevalence OR magnitude) AND (Antenatal OR Pregnancy OR prenatal OR women) AND (Depression OR depressive symptoms OR depressive disorder OR depressive) AND (Ethiopia). For the other two electronic databases (EMBASE and SCOPUS) we used database specific subject headings linked with the above terms and keywords used in PubMed. We also screened at the reference lists of the remaining papers to identify additional relevant studies to this review. This review protocol was written and presented according to PRISMA-P 2015 guidelines [39].

\section{Systematic review registration}

The protocol for this systematic review and meta-analysis was registered at PROSPERO International Prospective Register of Systematic Reviews 2017 (record ID=CRD42017076521, 06

December 2017)

Definition of concepts. In this review, institution base study refers to the study conducted in a clinical setting such as hospital and health centers whereas the community-based study refers to the study conducted in non-clinical settings including household surveys or home interviews.

\section{Eligibility criteria}

Two investigators (GA and GT) independently screened the selected studies using their titles and abstracts before retrieval of full-text papers. We used prespecified inclusion criteria to further screen the full-text articles. Disagreements were discussed during a consensus meeting with a third reviewer (SS) for final selection of studies to be included in the systematic review and meta-analysis. 


\section{Inclusion criteria}

- Design type-observational studies

- Study participants-pregnant women

- Those articles published in the English language

- Studies that reported on either prevalence and/or determinants of antenatal depression

- Studies were done in Ethiopia

\section{Exclusion criteria}

- Studies dealing with depressive disorders in nonpregnant women

- Studies dealing with perinatal depressive disorders associated with the postpartum period

- We also excluded editorials, letters, reviews, commentaries, and interventional studies

- Studies whose full data was not accessible even after requests from the authors were also excluded

- We also excluded duplicate studies

\section{Data extraction}

Two investigators (GA and GT) independently extracted the data from the studies included in our analysis as recommended by PRISMA guidelines [39]. The data was extracted using a standard data extraction form. The following information was extracted from the selected studies: first author's name, study population, the type of study design, sample size, study setting, data collection instrument, year of publication, tools used for assessing outcome, measures of effect (odds ratio (OR)), confounding variables, and the possible associated factors results.

\section{Data synthesis and quality assessment}

In this study, all the analysis was conducted using comprehensive meta-analysis software version3 [40]. We pooled the overall prevalence estimates of AND by a random effect meta-analysis [41]. We examined the heterogeneity of effect size using Q statistic and the $\mathrm{I}^{2}$ statistics[41]. The Q-test measures whether the observed effect size is considerably different from one another than expected by chance. When $\mathrm{Q}$ test is higher than the degree of freedom it indicates significant heterogeneity (also supplemented by $\mathrm{P}$ value). The $\mathrm{I}^{2}$ statistics assess the proportion of total variance across the included studies contributed to the observed heterogeneity. In this study, the $\mathrm{I}^{2}$ statistic value of zero indicates true homogeneity, whereas the value 25,50 and $75 \%$ were considered to represent true, low, medium and high, respectively [42]. For the data identified as heterogeneous, we conducted our analysis by random-effects model analysis. When statistical pooling is not possible, non-pooled data was presented in table form.

We evaluated the quality of selected studies by a modified version of the Newcastle-Ottawa Scale (NOS) [43]. As recommended by NOS scale, we evaluated the included studies by the following domains: first, sample size and representativeness; second, comparability between participants; third, ascertainment of antenatal depressive symptoms, and statistical quality. To 
evaluate the agreement between the two reviewers we used actual agreement and agreement beyond chance (unweighted Kappa).

We also conducted a leave one- out sensitivity analysis to appraise the main studies that exert a most important impact on between-study heterogeneity.

Moreover, to further identify the possible source of heterogeneity among the studies we conducted subgroup and sensitivity analysis based on the stages of pregnancy, the instrument used to measure AND, the study setting, and the quality of the included studies.

Finally, the funnel plot and Egger's regression tests were used to measure the presence of substantial publication bias.

\section{Results}

\section{Identification of studies}

A total of 193 studies were identified using electronic search engine and strategies. Of these, 186 were excluded during the evaluation of duplicate and titles as they did not meet the inclusion criteria (Fig 1). The evaluation of abstract resulted in the exclusion of a further one article. Therefore, a full-text of 6 studies were retrieved for further assessment and one of these was excluded.

\section{Characteristics of included studies}

Five papers were included in this systematic review and meta-analysis [32-36]. The characteristics of the included articles were shown in Table 1 . Selected studies were conducted between 2013 and 2017. All studies included in the final analysis used a cross-sectional study design. Two of the studies used community samples $[32,36]$ and three of the studies used samples from the institution [33-35]. Regarding the instrument used measure AND, two of the studies used beck depression inventory (BDI) $[33,34]$ whereas three of the studies used Edinburgh Postnatal Depression Scale (EPDS) [32, 35, 36] to measure antenatal depression (Table 1).

\section{Quality of included studies}

In our quality evaluation, we found that all the included studies were of reputable methodological quality (NOS) score ranges between 8 to 9 from a total 9-point NOS score). Investigators reached an agreement that the risk of selection, ascertainment and non-response bias was low. A moderate or almost perfect agreement was found between investigators regarding the level of bias for the studies included in the final analysis (Kappa statistic range 0.50-1 (S2 Table).

\section{The results of a pooled meta-analysis}

Prevalence of AND. Five studies were included in the meta-analysis, resulting in a reasonable samples size [32-36] (Table 1). From those studies, the pooled prevalence of AND in Ethiopia was $21.28 \%$ (95\% CI;15.96-27.78). We found a significant heterogeneity among the five studies $\left(I^{2}=90.61 \% ; \mathrm{Q}=42.61, \mathrm{df}=4\right.$, variance $\left.=0.014, \mathrm{p}<0.001\right)$. (Fig 2).

The subgroup analyses prevalence of AND during the three trimesters of pregnancy. We conducted a subgroup analysis of studies which provided information regarding the prevalence of AND in each trimester of pregnancy. Of the totally included studies, four studies measured the prevalence of AND in the second and third trimesters of pregnancy, whereas only two studies estimated the prevalence of AND in the first trimester of pregnancy. The results of the subgroup analysis showed that the pooled prevalence of AND was highest in the third trimester of pregnancy at $32.10 \%(95 \% \mathrm{CI} ; 12.93 \%, 60.07 \%)$, and it was $19.13 \% 19.13 \%(4.16 \%$, 


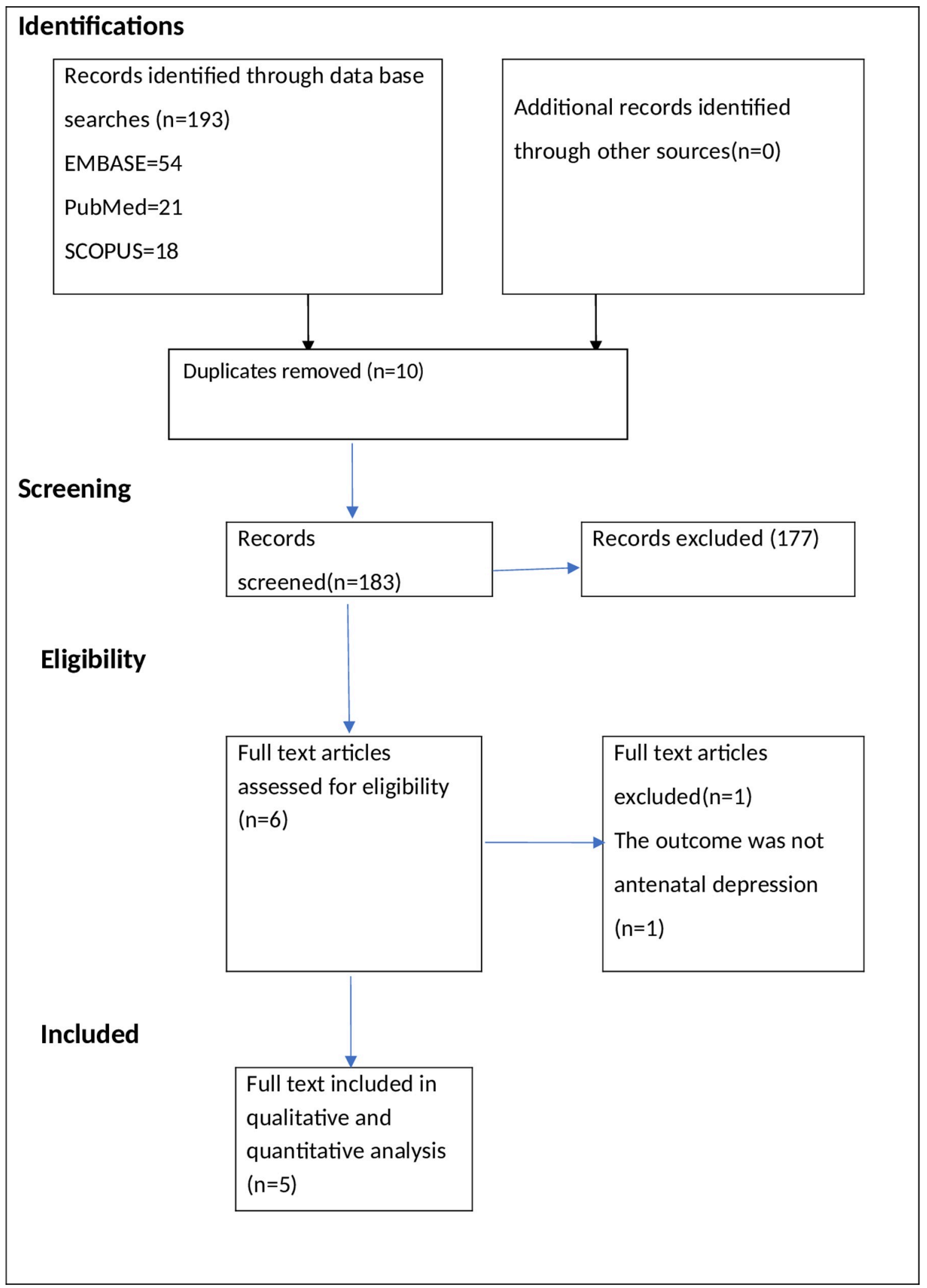

Fig 1. PRISMA flowchart of review search.

https://doi.org/10.1371/journal.pone.0211764.g001 
Table 1. Distribution of studies on antenatal depression included in the qualitative analysis based on year, study design, setting, sample size, instrument, and prevalence.

\begin{tabular}{|c|c|c|c|c|c|c|c|}
\hline $\begin{array}{l}\text { Author(year) (reference } \\
\text { number) }\end{array}$ & Study design & Setting & $\begin{array}{l}\text { Sample } \\
\text { size }\end{array}$ & Tool & Prevalence & $\begin{array}{l}\text { response } \\
\text { rate }\end{array}$ & Sampling \\
\hline $\begin{array}{l}\text { Bisetegn TA. et.al (2016) } \\
\text { [29] }\end{array}$ & $\begin{array}{l}\text { Cross sectional } \\
\text { study }\end{array}$ & Community based & 527 & EPDS & $11.8 \%$ & $97 \%$ & Cluster sampling \\
\hline $\begin{array}{l}\text { Ayele TA et. al. (2016) } \\
{[30]}\end{array}$ & $\begin{array}{l}\text { Cross sectional } \\
\text { study }\end{array}$ & Institution based & 388 & BDI & $23 \%$ & $92.82 \%$ & Systematic random sampling \\
\hline $\begin{array}{l}\text { Biratu, A.,et. al (2015) } \\
{[31]}\end{array}$ & $\begin{array}{l}\text { Cross sectional } \\
\text { study }\end{array}$ & $\begin{array}{l}\text { Institution-based } \\
\text { study }\end{array}$ & 393 & EPDS & 24.94 & $93.13 \%$ & Random sampling \\
\hline $\begin{array}{l}\text { Dibaba Y et.al (2013) } \\
\text { [33] }\end{array}$ & $\begin{array}{l}\text { Cross sectional } \\
\text { study }\end{array}$ & $\begin{array}{l}\text { Community-based } \\
\text { study }\end{array}$ & 622 & EPDS & $19.99 \%$ & $99 \%$ & $\begin{array}{l}\text { Census (as base line assessment for community- } \\
\text { based cohort study) }\end{array}$ \\
\hline $\begin{array}{l}\text { Mossie TB.et.al (2015) } \\
\text { [32] }\end{array}$ & $\begin{array}{l}\text { Cross sectional } \\
\text { study }\end{array}$ & Institution based & 196 & BDI & $31.1 \%$ & $93.8 \%$ & Systematic random sampling \\
\hline
\end{tabular}

Key: BDI: Beck depression inventory, EPDS: Edinburgh Postnatal Depression Scale

$56.31 \%)$ in the first trimester and $18.86 \%(95 \% \mathrm{CI} ; 11.51 \%, 28.87 \%)$ in the second trimester of pregnancy.

We found a considerable heterogeneity $\left(I^{2}=88.84 \% ; \mathrm{p}=0.003\right),\left(I^{2}=85.86 \% ; \mathrm{p}<0.001\right)$, and $\left(I^{2}=98.22 \% ; \mathrm{p}<0.001\right)$ for the first, second, and trimesters of pregnancy respectively (See Table 2).

Subgroup analysis of the prevalence of AND by the instrument used. We also conducted a subgroup analysis by the type of instrument used to measure AND. The pooled prevalence of AND was $26.48 \%$ (95\%CI; 19.09\%, 35.48\%) and 18.28\% (95\% CI;12.13-26.60) for studies conducted by using BDI and EPDS respectively. The heterogeneity was significant for both studies performed by BDI $\left(I^{2}=79.38 \%, \mathrm{Q}=4.85, \mathrm{df}=1, \mathrm{p}=0.028\right)$ as well as EPDS $\left(I^{2}=\right.$ 92.51\%, $\mathrm{Q}=26.72, \mathrm{df}=2 \mathrm{p}<0.001$ ). (See Table 2).

Subgroup analysis of the prevalence of AND by study setting. Finally, we conducted a subgroup analysis based on the study setting. The pooled prevalence estimates of AND was $15.50 \% \%$ (95\% CI;9.05-25. 27\%) for the studies conducted in the community setting and it was $25.77 \%$ (95\% CI 21.62-30.41) for the studies conducted in the institution-based setting. The heterogeneity was significant for community setting $I^{2}=92.73 \%, \mathrm{Q}=13.759, \mathrm{df}=1$, $\mathrm{p}<0.0001)$ as well as the institution-based setting $\left(I^{2}=59.39 \%, \mathrm{Q}=4.921, \mathrm{df}=2, \mathrm{p}=0.085\right)$. (See Table 2).

\section{Publication bias}

In the present study, no evidence of substantial publication bias was provided by the funnel plot and Egger's regression tests $(B=2.66, \mathrm{SE}=11.75, \mathrm{P}=0.84)$ for the prevalence of AND in Ethiopia (Fig 3).

\section{Sensitivity analysis}

We employed a leave-one-out sensitivity analysis to identify the potential source of heterogeneity in the analysis of the prevalence of AND in Ethiopia. The results of this sensitivity analysis showed that our findings were robust and not dependent on a single study. Our pooled estimated prevalence of AND varied between 19.93 (14.21-20.17) and 24.12 (25.5-30.84) after deletion of a single study (see Table 3).

To identify the source of heterogeneity we further conducted sensitivity analysis by study setting, the instrument used to ascertain AND as well as the prevalence of AND in each 
Event Lower Upper

rate limit limit $p$-Value
Relative

weight

19.72

20.16

20.30

20.75

19.08
Mossie $2015 \quad 0.31120 .2503 \quad 0.3794 \quad 0.0000$

$\begin{array}{llll}0.2128 & 0.1596 & 0.2778 & 0.0000\end{array}$

Biratu $2015 \quad 0.2494 \quad 0.2091 \quad 0.2945 \quad 0.0000$

Dibaba 2013

$0.19940 .1698 \quad 0.2326$

0.0000

Bisetegn $20160.11760 .0928 \quad 0.1481 \quad 0.0000$

Avele 2016

$\begin{array}{llll}0.2268 & 0.1878 & 0.2711 & 0.0000\end{array}$

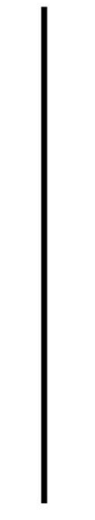

$-0.50$

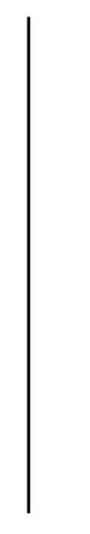

$-0.25$
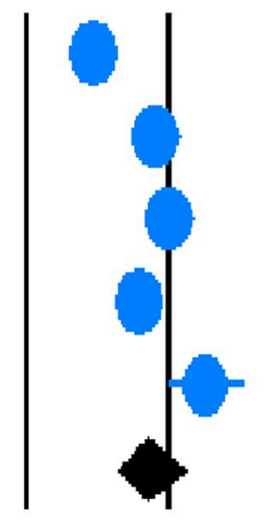

0.25

0.50

Keys: ${ }^{2}=90.61 \% ; Q=42.62 ; \mathrm{df}=4 ; \mathrm{P}<0.0001$ : Based on random effect analysis

Fig 2. The prevalence of AND in Ethiopia: a meta-analysis.

https://doi.org/10.1371/journal.pone.0211764.g002

trimester of pregnancy. When limiting our analysis to the prevalence of AND in each trimester of pregnancy, the prevalence of AND was highest in the third trimester of pregnancy (32.10), as compared to the prevalence estimate in the first $(19.13 \%)$ and second $(18.86 \%)$ trimesters of pregnancy, although the difference was not statistically significant $(P=0.544)$. (See Table 2$)$.

Moreover, when restricting our analysis to the prevalence of AND measured by using specific tools used to measure AND, the prevalence was highest in studies conducted using BDI (26.48\%) than studies conducted using EPDS (18.82\%). We found significant heterogeneity between the studies conducted using BDI and EPDs with a significant $\mathrm{p}$-value between the group variations $(\mathrm{P}=0.032)$. (See Table 2$)$.

Additionally, in our stratified analysis by the study setting, the prevalence of AND was greater in studies conducting in institution-based setting (25.78\%) than community-based setting $(15.50 \%)$, although the difference between the groups was not statistically significant $(\mathrm{p}=0.56)$. (See Table 2$)$. 
Table 2. Subgroup analysis of the prevalence of antenatal depression in Ethiopia based on random effect analysis.

\begin{tabular}{|c|c|c|c|c|c|c|}
\hline \multirow[t]{2}{*}{ Subgroup } & \multirow[t]{2}{*}{ Number of studies } & \multicolumn{2}{|l|}{ Estimates } & \multicolumn{2}{|c|}{$\begin{array}{l}\text { Heterogeneity across } \\
\text { the studies }\end{array}$} & \multirow[t]{2}{*}{ Heterogeneity between groups ( $P$ value } \\
\hline & & Prevalence (\%) & 95\% Confidence interval & $\mathrm{I}^{2}(\%)$ & $P$ value & \\
\hline \multicolumn{7}{|l|}{ Trimester of pregnancy } \\
\hline First & 2 & 19.13 & $4.16-56.31$ & 88.84 & $\mathrm{P}=003$ & 0.544 \\
\hline Second & 4 & 18.86 & $11.51-28.87$ & 85.86 & $\mathrm{P}<001$ & \\
\hline Third & 4 & 32.10 & $12.93-60.07$ & 98.22 & $\mathrm{P}<0001$ & \\
\hline \multicolumn{7}{|l|}{ Instrument use } \\
\hline BDI & 2 & $26.48 \%$ & $19.09-35.48$ & 79.38 & $\mathrm{P}=0.028$ & \multirow[t]{2}{*}{$P=0.032$} \\
\hline EPDS & 3 & 18.82 & $12.13-26.60$ & 92.51 & $\mathrm{P}<001$ & \\
\hline \multicolumn{7}{|l|}{ Setting } \\
\hline Community based & 2 & 15.50 & $9.05-25.27$ & 92.73 & $\mathrm{P}<001$ & \multirow[t]{2}{*}{0.056} \\
\hline Institution based & 3 & 25.78 & $21.62-30.41$ & 59.39 & $\mathrm{P}=0.085$ & \\
\hline \multicolumn{7}{|l|}{ NOS scale quality score } \\
\hline Above 8 & 2 & 16.50 & $14.50-18.80$ & 92.49 & $\mathrm{P}<001$ & 0.051 \\
\hline Less than or equal to 8 & 3 & 25.40 & $22.80-28.20$ & 59.34 & $\mathrm{P}=0.085$ & \\
\hline
\end{tabular}

https://doi.org/10.1371/journal.pone.0211764.t002

Finally, when restricting our analysis by NOS quality score, the prevalence of AND was greater in low quality score studies (NOS score less than or equal to 8 ) studies (25.40\%) than high quality score studies (NOS score above 8$)(16.50 \%)$, although the difference between the groups was not statistically significant $(\mathrm{p}=0.051)$. (See Table 2$)$.

\section{Narrative review}

Determinants of AND. In this section, we qualitatively analyzed the factors that were associated with an increased risk of AND in Ethiopia form the included five studies [32-36] (Table 4). In general, we observed that the level of adjustment for the possible confounding factors that are responsible for a greater risk of AND was inconsistent in Ethiopian studies. For example, in our review, we found that the association between the previous history of depression and AND was assessed only by two studies $[32,35]$. Similarly, only two studies measured the link between AND and unplanned pregnancy[32, 36], and being house wife's $[33,34]$. Moreover, the association between AND and other factors was assessed inconsistently (i.e. in each study different types of factors are measured as possible predisposing factors for AND). Therefore, it was difficult to combine and provide the pooled mean effects of factors associated with AND in Ethiopia.

In this study, the determinants of AND were categorized into the following three domains: sociodemographic factors (eight determinants), clinical and pregnancy-related factors (six determinants), and psychosocial and other factors (nine determinants). The overview of these factors including, the measures of effects, the strength of association and corresponding articles were presented in Table 4.

Sociodemographic factors. As summarized in Table 2, eight sociodemographic factors were significantly associated with an increased risk of AND [32-36]. The sociodemographic factors significantly associated with AND were low income (AOR $=5.12 \mathrm{CI} ; 1.42,18.48$ ) [34], being unmarried $(\mathrm{AOR}=4.07 \mathrm{CI} ; 1.18,14.04)$ [34], farmers $(\mathrm{AOR}=3.43 \mathrm{CI} ; 1.96-6.05)$ [36], and had debt $(\mathrm{AOR}=2.79 \mathrm{CI} ; 1.33,5.85)$. Furthermore, those who were housewife (AOR 2.57; $\mathrm{CI} ; 1.21 ; 5.46)[33]$, had food insecurity $(\mathrm{AOR}=4.60, \mathrm{CI} ; 2.75 ; 7.70)$ and employed 


\section{Funnel Plot of Standard Error by Logit event rate}

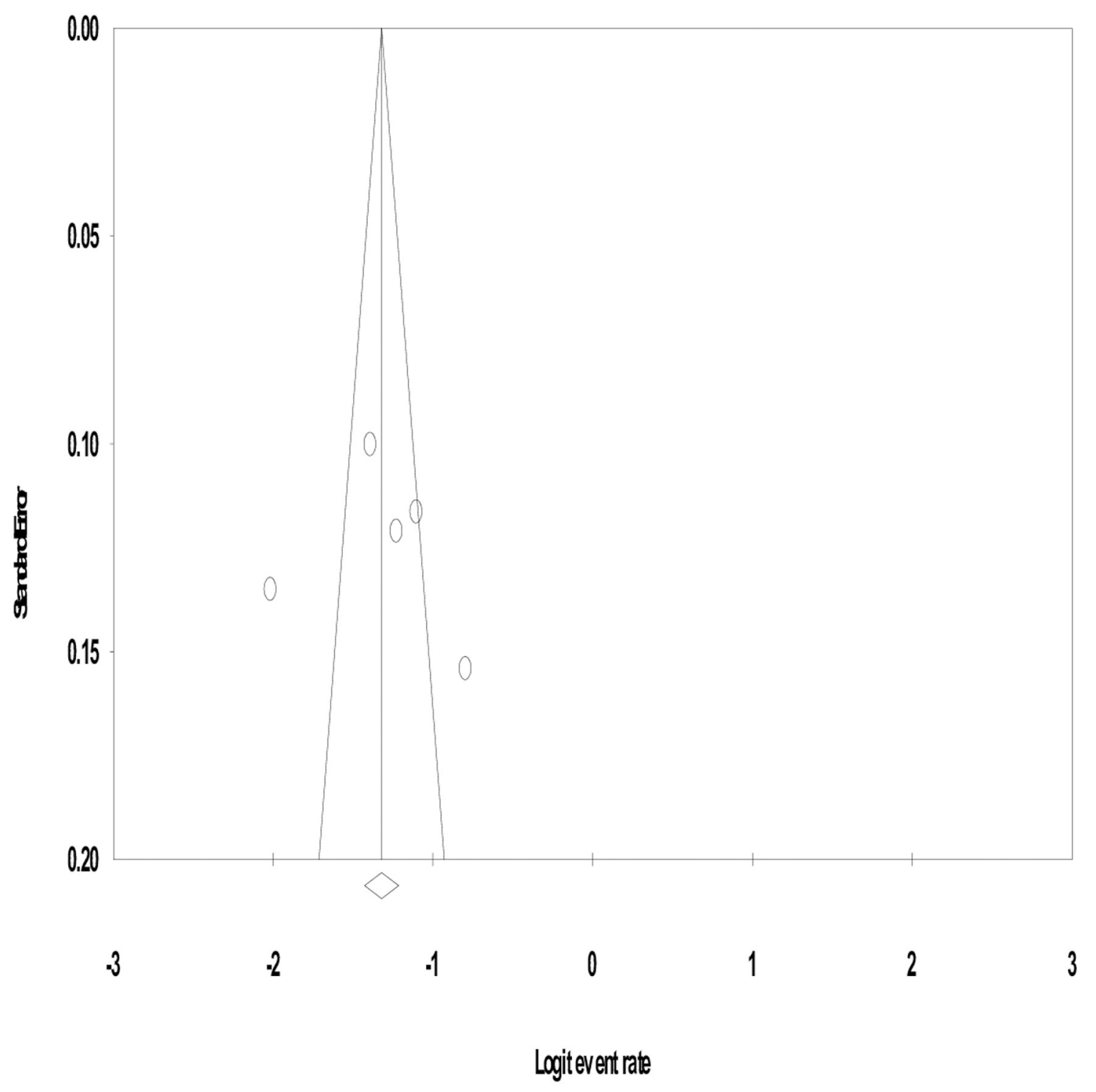

Fig 3. Funnel plot of risk of publication bias for the prevalence of antenatal depression in Ethiopia.

https://doi.org/10.1371/journal.pone.0211764.g003

$(\mathrm{AOR}=2.50, \mathrm{CI} ; 1.13-5.56$ ), and were in the age range 20-29 82 (AOR 0.18; CI; 0.07-0.49)

[33] were associated with a higher risk of developing AND[36].

Clinical and pregnancy-related factors. As illustrated in Table 4, six clinical and pregnancy-related factors were positively and significantly associated with a higher risk of AND in Ethiopia [32-36]. Those pregnant women who were nullipara (AOR $=4.74, \mathrm{CI} ; 1.58 ; 14.17$ [33], 
Table 3. Sensitivity analysis of prevalence for each study being removed at a time: Prevalence and $95 \%$ confidence interval of antenatal depression in Ethiopia.

\begin{tabular}{l|l|l}
\hline Study excluded & prevalence & 95\%CI \\
\hline Bisetegn TA. et.al (2016) [32] & 24.12 & $20.17-28.56$ \\
\hline Ayele TA et. al. (2016) [33] & 20.95 & $14.40-29.45$ \\
\hline Biratu, A.,et. al (2015) [35] & 20.42 & $14.21-28.46$ \\
\hline Dibaba Y et.al (2013) [36] & 21.65 & $14.62-30.84$ \\
\hline Mossie TB.et.al (2015) [34] & 19.33 & $14.33-25.56$ \\
\hline
\end{tabular}

Key. The analysis is based on the random effect model

https://doi.org/10.1371/journal.pone.0211764.t003

and had an unwanted pregnancy $\mathrm{AOR}=1.96, \mathrm{CI} ; 1.04-3.69)[36]$ were at a greater risk of developing AND.

Similarity, pregnant women who were in the third trimester of pregnancy $(\mathrm{AOR}=1.70, \mathrm{CI}$ $(1.07,2.72))[32]$ and those whose had complications during pregnancy $(\mathrm{AOR}=3.29 \mathrm{CI} ; 1.66$, 6.53) [32]) linked with higher risk of developing AND.

In addition, in this review those whose pregnancy was unplanned [35] and had the previous history of depression [32,35] were associated with a higher risk of developing AND.

Psychosocial and other factors. As indicated in Table 4, nine psychosocial and other factors were linked with increased risk of AND in Ethiopia. Those pregnant women who

Table 4. Characteristics of factors associated with antenatal depression in Ethiopia by their odds ratio, confidence interval strength of association, author and year.

\begin{tabular}{|c|c|c|c|c|}
\hline Factors & Odds ratio(AOR) & 95\% Confidence interval & Strength of association & Author, year \\
\hline History of still birth & 3.97 & $1.67-9.41$ & Strong, positive & Bisetegn, 2016 \\
\hline Complications during pregnancy & 3.29 & $1.66-6.53$ & Strong., positive & Bisetegn, 2016 \\
\hline Previous history of depression & 3.48 & $1.71-7.06$ & Strong, positive & Bisetegn, 2016 \\
\hline Having debt & 2.79 & $1.33-5.85$ & Moderate, positive & Bisetegn, 2016 \\
\hline Unplanned pregnancy & 2.39 & $1.20-4.76$ & Moderate, positive & Bisetegn, 2016 \\
\hline History of abortion & 2.57 & $1.005-6.61$ & Moderate, positive & Bisetegn, 2016 \\
\hline 3 rd trimester of pregnancy & 1.70 & $1.07-2.72$ & Moderate, positive & Bisetegn, 2016 \\
\hline Stressful life event & 2.04 & $1.01-4.11$ & Moderate, positive & Bisetegn, 2016 \\
\hline House wife & 2.57 & $1.21-5.46$ & Moderate, positive & Ayele, 2016 \\
\hline No ANC follow up & 11.98 & $4.73-30,33$ & Strong, positive & Ayele, 2016 \\
\hline Irregular ANC follow up & 11.43 & $3.68-35.49$ & Strong, positive & Ayele, 2016 \\
\hline Not satisfied by ANC follow up & 4.78 & $1.71-13.11$ & Strong, positive & Ayele, 2016 \\
\hline Age group 20-29 & 0.18 & $0.07-0.49$ & Strong, negative & Ayele, 2016 \\
\hline Unmarried & 4.07 & $1.18-14.04$ & Strong, positive & Mossie, 2017 \\
\hline Monthly income level $<1500$ Ethiopian birr & 5.12 & 1.42 & Strong, positive & Mossie, 2017 \\
\hline House wife & 4.24 & $1.38-13.03$ & Strong, positive & Mossie, 2017 \\
\hline Previous history of depression & 2.569 & $1.475-4.475$ & Moderate, positive & Biratu, 2015 \\
\hline Poor baby's father support & 1.89 & $1.064-3.358$ & Moderate, positive & Biratu, 2015 \\
\hline Unplanned pregnancy & 2.779 & $1.594-4.846$ & Moderate, positive & Biratu, 2015 \\
\hline Partner violence during pregnancy & 3.41 & $1.18-9.10$ & Strong, positive & Dibaba, 2013 \\
\hline Food insecurity & 4.60 & $2.75-7.70$ & Strong, positive & Dibaba, 2013 \\
\hline$\underline{\text { Farmer }}$ & 3.43 & $1.95-6.05$ & Strong, positive & Dibaba, 2013 \\
\hline High social support & 0.23 & $0.11-0.47$ & Strong, negative & Dibaba, 2013 \\
\hline Medium social support & 0.27 & $0.14-0.53$ & Strong, negative & Dibaba, 2013 \\
\hline Unwanted pregnancy & 1.96 & $1.04-3.69$ & Moderate, positive & Dibaba, 2013 \\
\hline
\end{tabular}

https://doi.org/10.1371/journal.pone.0211764.t004 
experienced partner violence during pregnancy [36], had lack of baby's father support during pregnancy) [35], had risky stressor [33] and those not satisfied by ANC follow-up) [33] were linked with a considerably higher risk of developing AND. Similarity, those who had medium social and high social support were less likely to have AND as compared to those who have low social support [36].

Moreover, those who have a history of abortion and stillbirth,[32], had irregular ANC follow-up pattern and no ANC follow up were more likely to have AND [33].

\section{Discussion}

In this systematic review and meta-analysis, we explored the prevalence and determinants of AND in Ethiopia. Five cross-sectional studies were included in the final analysis. Based on the meta-analysis a significant proportion (more than 1 in 5) of women had AND in Ethiopia. This shows that AND is a significant public health problem in Ethiopia. We also identified 23 determinants that were significantly associated with antenatal depression in Ethiopia.

In this study, the pooled prevalence of AND in Ethiopia was 21.28\% (95\% CI 15.96-27.78). These findings were consistent with the results from a recent meta-analysis that found pooled estimates for AND in the low and middle-income countries (LMICs) 25.3\% [11]. However, our results were lower than unpooled results from the studies conducted in some low and middle-income countries (LMICs), such as, $29 \%$ in Bangladesh [28], $39 \%$ in South Africa Cape Town, $38.5 \%$ [29] in South Africa KwaZulu-Natal[30] and $39.5 \%$, in Tanzania [31]. The results of this meta-analysis were higher than in studies conducted in other low and middleincome countries (LMICs), such as $20.2 \%$ in Brazil [26], $25 \%$ in Pakistan[27]. These differences might be due to the socioeconomic and cultural differences between the countries. The other possible reasons for the observed variation may be the use of a different instrument to assess antenatal depression. Moreover, the other obvious reason for the various might be the sample size, a collection of data from different settings (community and institution setting) as well as different study periods. This is because in the above studies the findings were based on the single study, but our findings were pooled prevalence estimates based on the five studies conducted in different setting and periods.

We also measured the pooled prevalence estimate of AND in each trimester of pregnancy. Our results found that the pooled prevalence estimate of AND was $19.13 \%(4.16 \%, 56.31 \%)$ in the first trimester of pregnancy. This finding was in line with a meta-analysis study done in developing countries which reported the pooled prevalence of AND 7.4\% in the first trimester of pregnancy. In the current study, the pooled prevalence estimates of AND were $18.86 \%$ $(11.51 \%, 28.87 \%)$ in the second trimester and $32.10 \%(12.93 \%, 60.07 \%)$ in the third trimester of pregnancy. These study findings are higher than a meta-analysis study done in developing countries which showed the pooled prevalence of AND 12.8\% and $12.0 \% 16.7 \%$ for the second, and third trimesters respectively[1]. These differences might be due to the sample size, socioeconomic and cultural differences between the countries.

In the current review, a subgroup analysis of the prevalence of AND by the instrument used showed that pooled prevalence result of AND of studies conducted by using beck depression inventory (BDI) was significantly higher in the results of the studies done using EPDS. The pooled prevalence of AND was $26.48 \%$ (95\%CI; $19.09 \%, 35.48 \%$ ) for the studies done by using beck depression inventory (BDI) and the pooled prevalence of AND was 18.28\% (95\% CI; 12.13-26.60) for the studies done by using EPDS. This difference might be due to BDI was not specifically prepared for detecting depression in pregnant population and it is also not validated in Ethiopia. Whereas EPDS is originally designed for assessing depression in pregnant 
and postpartum women and validated in Ethiopia. In addition, BDI has items used to assess somatic symptoms for depression which are usually common in pregnant women.

Subgroup analysis of the prevalence of AND by study setting, show that pooled prevalence result of AND of studies done in institution-based setting was significantly higher in the results of the studies done in the community setting. The pooled prevalence of AND was $15.50 \%$ (9.s \%CI, 9.05-25.27) for the studies done in institution-based setting and the pooled prevalence of AND was $25.77 \%$ (95\% CI 21.62-30.41) for the studies done in the community setting. This difference might be due to those who came to institution most likely have somatic symptoms and serious symptoms which are easily detected.

In our review we identified that factors which had robust scientific evidence as determining factor for AND in different worldwide studies, such as family history of depression, previous history of depression, substance use during pregnancy, any mental disorder during pregnancy, social support, unwanted pregnancy, stigma, partner violence during pregnancy, early pregnancy, stressful life events, complications during pregnancy, and economic status were either not included in the model or no assessed as confounding factors in each of the five included studies. Additionally, we observed that the level of adjustment for the possible confounding factors that are responsible for a greater risk of antennal depression was inconsistent in Ethiopian studies. For example, in our review, we found that the association between the previous history of depression and antenatal depression was assessed only by two studies [32, 35]. Similarly, only two studies measured the link between antenatal depression and unplanned pregnancy[32, 36], and being house wife's [33, 34]. Moreover, the association between AND and other factors was assessed inconsistently (i.e. in each study different types of factors are measured as possible predisposing factors for antenatal depression). Therefore, the association observed between AND and various factors reported in those included studies might be due to an inadequate level of adjustment for the possible confounding factors.

\section{Difference between the studies included in systematic review and meta- analysis}

The variations between the included studies resulted in an apparent heterogeneity in our meta-analysis. The instrument used to measure AND, the stage of pregnancy, the study setting, the sample size, and the study population varied on several characteristics which might have contributed for the observed difference in the magnitude of AND among the studies in Ethiopia. To further identify the possible source of heterogeneity in the estimates of AND, we conducted a leave-one-out analysis. The analysis showed that our findings were robust and not dependent on a single study.

In our sensitivity analysis, we found that the main cause of the observed variation in the prevalence of AND was found to be the instrument used to estimate AND. The prevalence was $26.8 \%$ as measured by BDI and $18.82 \%$ as measured by EPDS with significant p-value between the studies conducted using the above two instruments ( $\mathrm{p}$ value $=0.032$ ). The possible explanation for the remarkable difference in the prevalence of AND among the various measuring instrument (BDI and EPDS) might be due to variations in the sensitivity (the test's ability to correctly identify a participant with the disease as positive) and specificity (the test's ability to correctly label a participant without the disease as negative) of the instruments. Epidemiologic evidence showed that the use of BDI during pregnancy was associated with an apparent overestimation of the magnitude of depression during pregnancy due to the existing overlap between depressive and somatic symptoms during pregnancy [44]. Therefore, a considerably higher magnitude of AND in studies conducted using BDI than EPDS may be due to the overlap between BDI and somatic symptoms of pregnancy. The results of our study support the 
view that validation and use of the standard instrument for screening and diagnosis of depression during pregnancy.

However, the difference observed among the included studies depending on the trimesters of pregnancy, the study setting, as well as the quality score, was not significant. Nevertheless, the sample size and a small number of studies included in each subgroup in our sensitivity analysis need to be acknowledged.

Finally, in order to make the findings of our meta-analysis meaningful, we employed a random-effects model. It's widely held that the summary effect estimates in the random effect model are more conservative than fixed-effects summaries in epidemiologic metaanalysis.

\section{Strength and limitations}

This study has several strengths: First, we used a prespecified protocol for search strategy and data abstraction and conducted quality assessment two independent investigators to lessen the possible assessor bias; Second, we employed subgroup and sensitivity analysis based on study setting, instrument used, and trimester of pregnancy to identify the small study effect and the risk of heterogeneity in; third, The quality of included studies was evaluated by two authors. Nevertheless, our systematic review and meta-analysis has some limitations: first, in our small number of studies were included in our subgroup analysis which reduce the precision of the estimate; second, a considerable heterogeneity was identified among the studies; due to inconsistent adjustment and inclusion of factors determining antenatal depression we did only qualitative analysis for associated factors of antenatal depression.

\section{Conclusion and recommendations}

In summary, in our meta-analysis, the pooled prevalence of AND in Ethiopia was 21.28\% (95\% CI; 15.96-27.78). The pooled prevalence of AND was highest in the third trimester of pregnancy at $32.10 \%$ and it was $19.13 \%$ in the first trimester and $18.86 \%$ in the second trimester of pregnancy. In addition, the pooled prevalence of AND was $26.48 \%$ and $18.28 \%$ as measured by BDI and the EPDS, respectively. The pooled prevalence of AND was $15.50 \%$ for the studies conducted in the community setting and it was $25.78 \%$ for the studies conducted in the institution-based setting.

In this study, we observed that the level of adjustment for the possible confounding factors that are responsible for a greater risk of AND was inconsistent in Ethiopian studies.

Studies on the magnitude of antenatal depression and determinants in each trimester of pregnancy with representative sample size and studies focusing on scientifically plausible and consistent factors on AND are recommended.

Future epidemiologic studies focusing on specific age groups such as adolescents, young adults, and disadvantaged women as well as on women with unintentional pregnancy, experienced violence and those who are substance and drug users during pregnancy were warranted. Furthermore, longitudinal studies focusing on incidence and determinates of AND are recommended.

Attention needs to be given for the mental health of pregnant women and the government needs to consider the possible integration of mental health services with the existing antenatal care in Ethiopia.

Finally, future studies have to be conducted using a validated instrument to be used in pregnant women. 


\section{Supporting information}

S1 Table. PRISMA-P (Preferred Reporting Items for Systematic review and Meta-Analysis Protocols) 2015 checklist: Recommended items addressed in our systematic review and meta-analysis.

(DOC)

S2 Table. Summary of quality assessment and agreed level of bias and level of agreement on the methodological qualities of included studies in meta-analysis based on sampling, outcome, response rate and method of analysis.

(DOCX)

\section{Author Contributions}

Conceptualization: Getinet Ayano.

Data curation: Getinet Ayano.

Formal analysis: Getinet Ayano.

Methodology: Getachew Tesfaw, Shegaye Shumet.

Writing - original draft: Getinet Ayano.

Writing - review \& editing: Getinet Ayano.

\section{References}

1. Bennett HA, Einarson A, Taddio A, Koren G, Einarson TR. Prevalence of depression during pregnancy: systematic review. Obstetrics \& Gynecology. 2004; 103(4):698-709.

2. Kuhner C. [Mental disorders in pregnancy and postpartum: Prevalence, course, and clinical diagnostics]. Nervenarzt. 2016; 87(9):926-36. Epub 2016/07/28. https://doi.org/10.1007/s00115-016-0175-0 PMID: 27456195

3. Osborne LM, Monk C. Perinatal depression—the fourth inflammatory morbidity of pregnancy?: theory and literature review. Psychoneuroendocrinology. 2013; 38(10):1929-52. https://doi.org/10.1016/j. psyneuen.2013.03.019 PMID: 23608136

4. Rusner M, Berg M, Begley C. Bipolar disorder in pregnancy and childbirth: a systematic review of outcomes. BMC pregnancy and childbirth. 2016; 16(1):331. https://doi.org/10.1186/s12884-016-1127-1 PMID: 27793111

5. DiPietro JA. Maternal stress in pregnancy: considerations for fetal development. Journal of Adolescent Health. 2012; 51(2):S3-S8.

6. Van den Bergh BR, Mulder EJ, Mennes M, Glover V. Antenatal maternal anxiety and stress and the neurobehavioural development of the fetus and child: links and possible mechanisms. A review. Neuroscience \& Biobehavioral Reviews. 2005; 29(2):237-58.

7. Andersson L, Sundstrom-Poromaa I, Bixo M, Wulff M, Bondestam K, aStrom M. Point prevalence of psychiatric disorders during the second trimester of pregnancy: a population-based study. American journal of obstetrics and gynecology. 2003; 189(1):148-54. Epub 2003/07/16. PMID: 12861154.

8. Gavin NI, Gaynes BN, Lohr KN, Meltzer-Brody S, Gartlehner G, Swinson T. Perinatal depression: a systematic review of prevalence and incidence. Obstetrics and gynecology. 2005; 106(5 Pt 1):1071-83. Epub 2005/11/02. https://doi.org/10.1097/01.AOG.0000183597.31630.db PMID: 16260528.

9. Lee AM, Lam SK, Sze Mun Lau SM, Chong CS, Chui HW, Fong DY. Prevalence, course, and risk factors for antenatal anxiety and depression. Obstetrics and gynecology. 2007; 110(5):1102-12. Epub 2007/11/06. https://doi.org/10.1097/01.AOG.0000287065.59491.70 PMID: 17978126.

10. Melville JL, Gavin A, Guo Y, Fan MY, Katon WJ. Depressive disorders during pregnancy: prevalence and risk factors in a large urban sample. Obstetrics and gynecology. 2010; 116(5):1064-70. Epub 2010/10/23. https://doi.org/10.1097/AOG.0b013e3181f60b0a PMID: 20966690; PubMed Central PMCID: PMCPMC3068619. 
11. Gelaye B, Rondon M, Araya R, Williams MA. Epidemiology of maternal depression, risk factors, and child outcomes in low-income and middle-income countries. The lancet Psychiatry. 2016; 3(10):97382. https://doi.org/10.1016/S2215-0366(16)30284-X PubMed PMID: PMC5155709. PMID: 27650773

12. Evans J, Heron J, Francomb H, Oke S, Golding J. Cohort study of depressed mood during pregnancy and after childbirth. BMJ (Clinical research ed). 2001; 323(7307):257-60.

13. Felice E, Saliba J, Grech V, Cox J. Prevalence rates and psychosocial characteristics associated with depression in pregnancy and postpartum in Maltese women. Journal of affective disorders. 2004; 82 (2):297-301. https://doi.org/10.1016/j.jad.2003.11.011 PMID: 15488261

14. Jesse DE, Walcott-McQuigg J, Mariella A, Swanson MS. Risks and protective factors associated with symptoms of depression in low-income African American and Caucasian women during pregnancy. Journal of Midwifery \& Women's Health. 2005; 50(5):405-10.

15. Josefsson A, Berg G, Nordin C, Sydsjö G. Prevalence of depressive symptoms in late pregnancy and postpartum. Acta obstetricia et gynecologica Scandinavica. 2001; 80(3):251-5. PMID: 11207491

16. Johanson R, Chapman G, Murray D, Johnson I, Cox J. The North Staffordshire Maternity Hospital prospective study of pregnancy-associated depression. Journal of Psychosomatic Obstetrics \& Gynecology. $2000 ; 21(2): 93-7$.

17. Lee DT, Chan SS, Sahota DS, Yip AS, Tsui M, Chung TK. A prevalence study of antenatal depression among Chinese women. Journal of affective disorders. 2004; 82(1):93-9. https://doi.org/10.1016/j.jad. 2003.10.003 PMID: 15465581

18. Adewuya AO, Ola BA, Aloba OO, Dada AO, Fasoto OO. Prevalence and correlates of depression in late pregnancy among Nigerian women. Depression and anxiety. 2007; 24(1):15-21. https://doi.org/10. 1002/da.20221 PMID: 16845663

19. Glasser S, Tanous M, Shihab S, Goldman N, Ziv A, Kaplan G. Perinatal depressive symptoms among Arab women in northern Israel. Maternal and child health journal. 2012; 16(6):1197-205. https://doi.org/ 10.1007/s10995-011-0845-2 PMID: 21735141

20. Marcus SM, Flynn HA, Blow FC, Barry KL. Depressive symptoms among pregnant women screened in obstetrics settings. Journal of Women's Health. 2003; 12(4):373-80. https://doi.org/10.1089/ 154099903765448880 PMID: 12804344

21. Rubertsson $C$, Wickberg $B$, Gustavsson $P$, Rådestad I. Depressive symptoms in early pregnancy, two months and one year postpartum-prevalence and psychosocial risk factors in a national Swedish sample. Archives of women's mental health. 2005; 8(2):97-104. https://doi.org/10.1007/s00737-005-00788 PMID: 15883652

22. Wissart J, Parshad O, Kulkarni S. Prevalence of pre-and postpartum depression in Jamaican women. BMC Pregnancy and Childbirth. 2005; 5(1):15.

23. Black MM, Baqui AH, Zaman $\mathrm{K}$, McNary SW, Le K, Arifeen SE, et al. Depressive symptoms among rural Bangladeshi mothers: implications for infant development. Journal of Child Psychology and Psychiatry. 2007; 48(8):764-72. https://doi.org/10.1111/j.1469-7610.2007.01752.x PMID: 17683448

24. Rahman A, Iqbal Z, Bunn J, Lovel H, Harrington R. Impact of maternal depression on infant nutritional status and illness: a cohort study. Archives of general psychiatry. 2004; 61(9):946-52. https://doi.org/ 10.1001/archpsyc.61.9.946 PMID: 15351773

25. Mathers CD, Loncar D. Projections of global mortality and burden of disease from 2002 to 2030. PLoS medicine. 2006; 3(11):e442. https://doi.org/10.1371/journal.pmed.0030442 PMID: 17132052

26. Faisal-Cury A, Menezes $P$, Araya R, Zugaib M. Common mental disorders during pregnancy: prevalence and associated factors among low-income women in São Paulo, Brazil. Archives of women's mental health. 2009; 12(5):335. https://doi.org/10.1007/s00737-009-0081-6 PMID: 19468824

27. Rahman A, lqbal Z, Harrington R. Life events, social support and depression in childbirth: perspectives from a rural community in the developing world. Psychological medicine. 2003; 33(7):1161-7. PMID: 14580070

28. Nasreen HE, Kabir ZN, Forsell Y, Edhborg M. Prevalence and associated factors of depressive and anxiety symptoms during pregnancy: a population based study in rural Bangladesh. BMC women's health. 2011; 11(1):22.

29. Hartley M, Tomlinson M, Greco E, Comulada WS, Stewart J, Le Roux I, et al. Depressed mood in pregnancy: prevalence and correlates in two Cape Town peri-urban settlements. Reproductive health. 2011 $8(1): 9$.

30. Manikkam L, Burns JK. Antenatal depression and its risk factors: an urban prevalence study in KwaZulu-Natal. SAMJ: South African Medical Journal. 2012; 102(12):940-4. https://doi.org/10.7196/samj. 6009 PMID: 23498042 
31. Kaaya S, Mbwambo J, Kilonzo G, Van Den Borne H, Leshabari M, Fawzi MS, et al. Socio-economic and partner relationship factors associated with antenatal depressive morbidity among pregnant women in. Tanzania journal of health research. 2010; 12(1):23-35. PMID: 20737826

32. Bisetegn TA, Mihretie G, Muche T. Prevalence and Predictors of Depression among Pregnant Women in Debretabor Town, Northwest Ethiopia. PLOS ONE. 2016; 11(9):e0161108. https://doi.org/10.1371/ journal.pone.0161108 PMID: 27618181

33. Ayele TA, Azale T, Alemu K, Abdissa Z, Mulat H, Fekadu A. Prevalence and Associated Factors of Antenatal Depression among Women Attending Antenatal Care Service at Gondar University Hospital, Northwest Ethiopia. PLOS ONE. 2016; 11(5):e0155125. https://doi.org/10.1371/journal.pone.0155125 PMID: 27153193

34. Mossie TB, Sibhatu AK, Dargie A, Ayele AD. Prevalence of Antenatal Depressive Symptoms and Associated Factors among Pregnant Women in Maichew, North Ethiopia: An Institution Based Study. Ethiopian Journal of Health Sciences. 2017; 27(1):59-66. PubMed PMID: PMC5390229. PMID: 28458491

35. Biratu A, Haile D. Prevalence of antenatal depression and associated factors among pregnant women in Addis Ababa, Ethiopia: a cross-sectional study. Reproductive Health. 2015; 12(1):99. https://doi.org/ 10.1186/s12978-015-0092-x PMID: 26514827

36. Dibaba Y, Fantahun M, Hindin MJ. The association of unwanted pregnancy and social support with depressive symptoms in pregnancy: evidence from rural Southwestern Ethiopia. BMC Pregnancy and Childbirth. 2013; 13(1):135. https://doi.org/10.1186/1471-2393-13-135 PMID: 23800160

37. Ajinkya S, Jadhav PR, Srivastava NN. Depression during pregnancy: Prevalence and obstetric risk factors among pregnant women attending a tertiary care hospital in Navi Mumbai. Industrial psychiatry journal. 2013; 22(1):37. https://doi.org/10.4103/0972-6748.123615 PMID: 24459372

38. Reviews UoYCf, Dissemination. Systematic reviews: CRD's guidance for undertaking reviews in health care: University of York, Centre for Reviews \& Dissemination; 2009.

39. Moher D, Shamseer L, Clarke M, Ghersi D, Liberati A, Petticrew M, et al. Preferred reporting items for systematic review and meta-analysis protocols (PRISMA-P) 2015 statement. Systematic reviews. 2015; 4(1):1.

40. Borenstein $\mathrm{M}$, Hedges L, Higgins J, Rothstein $\mathrm{H}$. Comprehensive meta-analysis version 2. Englewood, NJ: Biostat. 2005; 104.

41. Borenstein M, Hedges LV, Higgins J, Rothstein HR. A basic introduction to fixed-effect and randomeffects models for meta-analysis. Research synthesis methods. 2010; 1(2):97-111. https://doi.org/10. 1002/jrsm.12 PMID: 26061376

42. Higgins JP, Thompson SG, Deeks JJ, Altman DG. Measuring inconsistency in meta-analyses. BMJ: British Medical Journal. 2003; 327(7414):557. https://doi.org/10.1136/bmj.327.7414.557 PMID: 12958120

43. Stang A. Critical evaluation of the Newcastle-Ottawa scale for the assessment of the quality of nonrandomized studies in meta-analyses. European journal of epidemiology. 2010; 25(9):603-5. https://doi. org/10.1007/s10654-010-9491-z PMID: 20652370

44. Curzik D, Begic NJ. The utility of BDI-II in assessment of pre- and postpartum depression symptoms and their relation to labor pain. Psychiatr Danub. 2012; 24(2):167-74. Epub 2012/06/19. PMID: 22706415. 